\title{
Bilateral decompressive surgery in lumbar spinal stenosis associated with spondylolisthesis: unilateral approach and use of a microscope and tubular retractor system
}

\author{
Sylvain Palmer, M.D., Robert Turner, M.D., and Rosemary Palmer, R.N. \\ Mission Hospital Regional Medical Center, Mission Viejo, California
}

\begin{abstract}
Object. The objective of this study was to assess the feasibility and efficacy of treating spondylolisthesis-related spinal stenosis via unilateral approach bilateral decompression in which METRX-MD instrumentation is placed.

Methods. Eight consecutive patients with spinal stenosis underwent bilateral decompressions via a unilateral approach in which METRx-MD instrumentation was placed. The procedures were performed on an outpatient basis after induction of general anesthesia. The patients underwent preoperative and 3-month postoperative plain radiography in which flexion-extension x-ray films were obtained. Preoperative and postoperative magnetic resonance imaging was also performed. All radiographs and neuroimages were read by a single radiologist blinded to the clinical results.

Eight vertebral levels in the eight patients were sugically decompressed (in one patient an additional level of nonspondylolisthesis-related stenosis was decompressed). The mean operative time was 92 minutes and the mean blood loss was $33 \mathrm{ml} / \mathrm{level}$. Preoperatively stenosis was severe in five patients, moderate/severe in two, and moderate in one; postoperatively stenosis was absent in five, mild in two, and mild/moderate in one. Motion was detected on flexion-extension radiographs in three patients, but on early (3-month) postoperative radiographs there was no evidence of progression.

Conclusions. By following the authors' procedure, minimally invasive bilateral decompression of acquired spinal stenosis associated with spondylolisthesis can be successfully performed on an outpatient basis, with reasonable operative times, minimal blood loss, and acceptable morbidity.
\end{abstract}

\section{KEY WoRDS • spinal stenosis • spondylolisthesis • laminotomy • minimally invasive surgery $\bullet$ outpatient surgery}

In 1911 Bailey and Casamajor ${ }^{4}$ extensively described spinal stenosis as a cause of neural compression. Surgical treatment of spinal stenosis was undertaken as early as $1900 .{ }^{22}$ Traditional treatment of spinal stenosis has involved wide laminectomy and undercutting of the medial facet with foraminotomy. ${ }^{26}$ More limited decompressive procedures including bilateral foraminotomies ${ }^{3,24,27}$ and unilateral approaches to bilateral decompression ${ }^{7,14,20}$ have been shown to be effective.

Minimally invasive procedures involving chemical, ${ }^{23}$ mechanical, ${ }^{16,19}$ laser, ${ }^{5}$ and endoscopic techniques ${ }^{10,16,19}$ have been applied in spine surgery. Foley and Smith ${ }^{10}$ have popularized the microendoscopic discectomy technique for disc surgery. This technique has further evolved with the introduction of a microendoscopic tubular retractor system for microdiscectomy (METRx-MD) that improves compatibility with the operative microscope. The extension of the METRx-MD placement-related techniques to intraspinal pathological entities other than herniated discs has evolved, and surgeons are increasingly familiar and comfortable with such a technique. We implanted the METRx-MD instrumentation via a unilateral

Abbreviations used in this paper: $\mathrm{CT}=$ computerized tomography; $\mathrm{MR}=$ magnetic resonance; VAS = visual analog scale. approach after performing bilateral decompression of acquired spinal stenosis in patients with Grade I degenerative spondylolisthesis.

\section{CLINICAL MATERIAL AND METHODS}

\section{Patient Population}

The first eight consecutive patients with spinal stenosis and Grade I spondylolisthesis to undergo bilateral decompression via a unilateral approach for and placement of METRx-MD instrumentation are included in this report. A single surgeon performed all surgeries. A total of eight spondylolisthetic levels were decompressed (in one patient an additional stenotic level, not involved with spondylolisthesis, was decompressed). Two discectomies were performed at the stenotic/spondylolisthetic levels. All operations were planned as outpatient procedures; however, one patient was admitted for 48 hours after suffering a duratomy. General anesthesia was induced in all patients; however, a local spinal anesthestic has been used successfully in several subsequent patients.

The mean patient age was 70.5 years (range 49-83 years). There were seven women and one man. The duration of symptoms was 44 months with a mean of 19 months. There were no smokers. Back pain was present in 
seven, claudication in three, radiculopathy in eight, reflex abnormalities in five, motor abnormalities in three, sensory abnormalities in four, and bowel and bladder abnormalities in one.

\section{Preoperative and Postoperative Assessments}

All patients underwent pre- and postoperative MR imaging studies. A single radiologist (R.T.), blinded to the clinical results of decompression, reviewed all pre- and postoperative studies. The $\mathrm{T}_{2}$-weighted axial MR images were graded with regard to the degree of stenosis: 0 , none; 1, mild; 2, mild/moderate; 3, moderate; 4, moderate/severe; and 5, severe (Fig. 1). In all patients preoperative and in seven patients 3-month postoperative lumbar flexion-extension radiographs were obtained. These were reviewed for evidence of instability and/or progression of spondylolisthesis. Our office nurse (R.P.) interviewed all patients 4 to 7 months postoperatively to assess their clinical progress, using a basic questionnaire, which included a VAS for pain developed by $\mathrm{PhDx}$ for spinal surgery.

\section{Surgical Procedure}

Patients were prepared and positioned as for standard laminectomy. The procedure is a modification of the microendoscopic discectomy procedure previously described in detail by Foley and Smith. ${ }^{10}$ The level of interest was localized using fluoroscopy, and an 18-mm paramedian skin incision was then made. The muscle was sequentially dilated, and an 18-mm working channel, of the shortest length that would allow adequate depth of access (usually 50 or $60 \mathrm{~mm}$ ), was placed. The operating microscope was moved into the field and the laminar edge

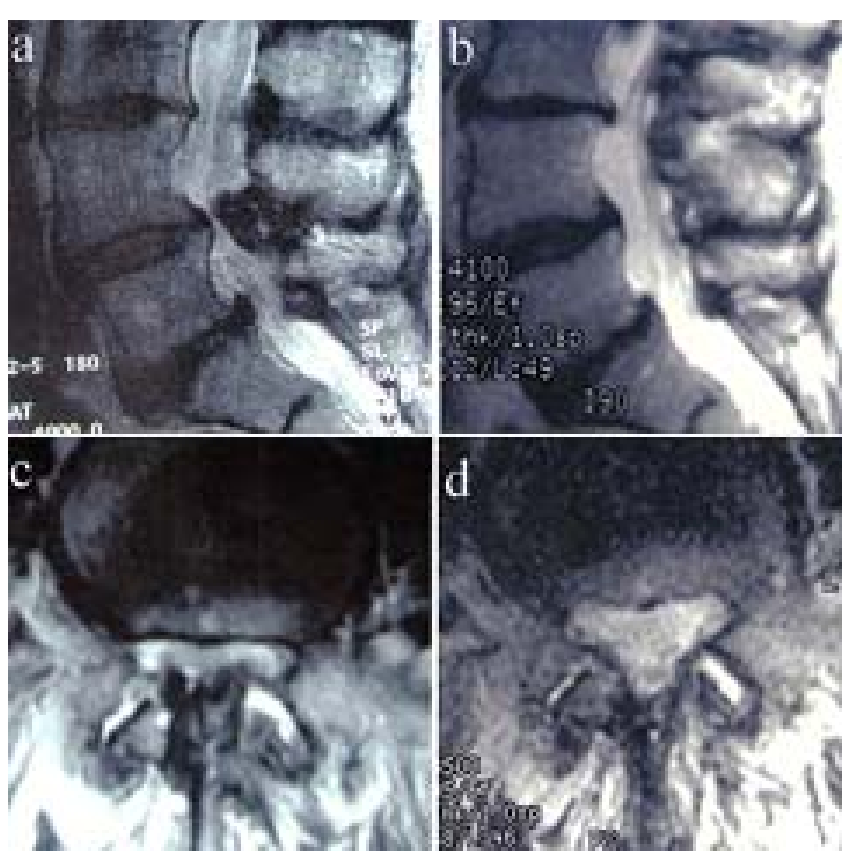

Fig. 1. Preoperative and postoperative $\mathrm{T}_{2}$-weighted $\mathrm{MR}$ images obtained in a patient undergoing L4-5 decompression. Preoperative (a) and postoperative (b) sagittal images, and pre- (c) and postoperative (d) axial images. was identified. A laminotomy was performed, extending cephalad to the insertion of the ligamentum flavum on the inferior surface of the superior lamina (to ensure adequate resection of ligamentous compressive elements) and caudally to include a smaller portion of the superior aspect of the inferior lamina, thus exposing the pedicle. Partial resection of the medial facet complex was performed as necessary to obtain an adequate decompression of the lateral recess and the foramina.

The working channel was then angled medially, exposing the anterior aspect of the spinous process, which was then removed using a Midas Rex drill (Fig. 2). This exposed the lateral recess on the contralateral side where residual lamina and ligamentum flavum could be resected using the drill, Kerrison punches, and curettes. The angle of approach was the same as that commonly undertaken during an open laminectomy, which allows undermining of the contralateral facets; thus, the anatomy is familiar to most spine surgeons. Satisfactory decompression of the lateral recess and foramina was achieved under direct visualization. Palpation of the pedicle and foramina, with a blunt ball-tipped nerve hook, ensured correct orientation and complete decompression (Fig. 3).

Depomedrol (80 mg) was instilled in the epidural space (unless duratomy had occurred) and bupivacaine $0.25 \%$ with epinephine 1:200,000 was infiltrated into the soft tissues. The incision was closed in layers by using Vicryl followed by placing Steristrips and a bioocclusive dressing or, more recently, Durmabond on the skin edge. The patients were encouraged to use ice postoperatively and were given only oral analgesic medication (usually hydrocodone). Patients were placed in a chair back brace for 6 weeks as a precautionary measure regardless of whether stablility was demonstrated preoperatively on flexionextension lateral radiographs. Physical therapy was initi-

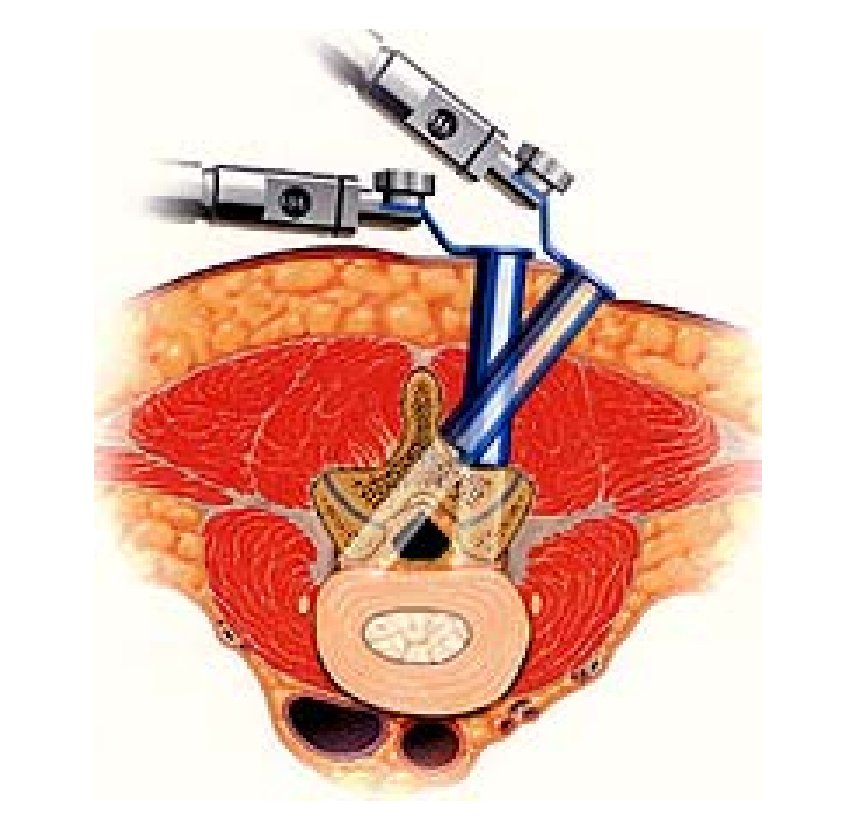

Fig. 2. Illustration showing the tubular retractor in vertical position and in medially angulated position to access the contralateral side. 


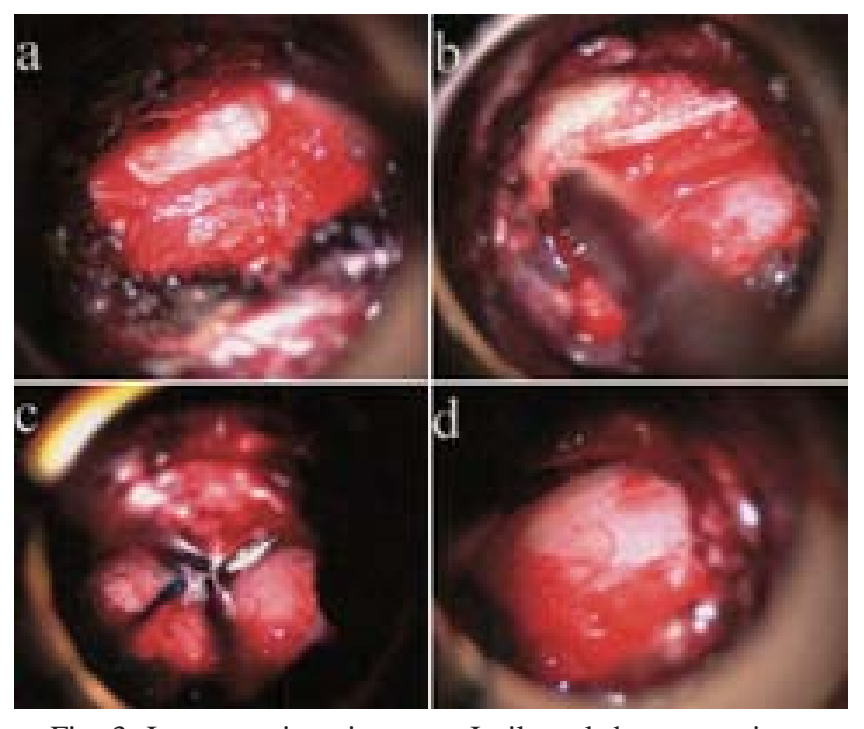

Fig. 3. Intraoperative views. a: Ipsilateral decompression exposing ipsilateral synovial cyst. b: Excising contralateral ligamentum flavum with a Kerrison punch. c: Blunt nerve hook on contralateral pedicle. d: Bilaterally decompressed dural sac.

ated at 6 weeks and thereafter increased activities were encouraged.

\section{Sources of Supplies and Equipment}

The METRx-MD system is manufactured by Medtronics (Memphis, TN). The basic questionnaire used to determine patients' postoperative satisfaction was produced by $\mathrm{PhDx}$ (Albuquerque, NM). We obtained the Midas Rex drill (model TDQ 130D) from Medtronics, the Vicryl and Durmabond from Johnson and Johnson (Somerville, NJ), and the Steristrips from 3M Corporation (St. Paul, MN).

\section{RESULTS}

A total of eight stenotic/spondylolisthetic levels were decompressed in the eight patients. There were seven single-level procedures and one two-level procedure (in the patient with a second stenotic level unrelated to spondylolistheses): L4-5 in six, L3-4 in one, and L5-S1 in one patient. Two patients underwent concomitant discectomies at the index level. The mean operative time was 92 minutes/level, and the mean estimated blood loss was 33 $\mathrm{ml}$. There was one duratomy that was not primarily repaired but covered with gelfoam. The patient was admitted for 48 hours of bedrest and did not experience adverse consequences. There were no neurological injuries and no infections.

Preoperatively stenosis was severe in five patients, moderate/severe in two, and moderate in one. Postoperatively stenosis was absent in five, mild in two, and mild/ moderate in one. The five patients in whom no residual stenosis was observed postoperatively, preoperative stenosis was severe in two, moderate/severe in two, and moderate in one patient. In the two cases in which postoperative stenosis was mild, it had formerly been graded as severe. The postoperative mild/moderate had been severe stenosis preoperatively (Fig. 4). One patient has required a subsequent surgery for a herniated nucleus pulposus that developed at a level different from the original surgically treated level. After a minimum 2-year follow-up period, no patient has required reexploration of the stenotic level or secondary fusion.

Degenerative spondylolisthesis (Grade I) was observed at eight different levels in the eight patients. Seven patients underwent pre- and postoperative flexion-extension radiography (in the eighth no increase in subluxation was demonstrated on her postoperative MR imaging study). Abnormal motion in the sagittal plane was observed on the preoperative $\mathrm{X}$-ray films in three patients. No increase in the degree of subluxation or the amount of motion was evident on postoperative $\mathrm{x}$-ray films in any patient.

Analysis of the postoperative responses in the questionnaire revealed that $88 \%$ of patients thought it was definitely or mostly true that they could do the things they thought they would be able to do postoperatively and $63 \%$ thought it was definitely or mostly true that, all things considered, they would have the surgery again (Fig. 5). At the time of the postoperative interview, $81 \%$ of patients required no pain medications for back pain and $88 \%$ required no pain medications for leg pain. The mean preoperative VAS pain score was 7.6 and the average postoperative VAS pain score was 2 (Fig. 6).

\section{DISCUSSION}

Many authors have challenged the traditional treatment of spinal stenosis in which wide laminectomy and partial or complete facetectomy was performed. A variety of less extensive resections have been proposed in an attempt to preserve normal spinal anatomy. ${ }^{3,7,14,18,20,24,27}$ Although the goal of limited resection is to lessen the risk of postoperative spondylolisthesis, it is important not to compromise the neural decompression in one's effort to be minimally invasive. The primary goal of the procedure is to obtain an adequate decompression of the neural elements. An ad-

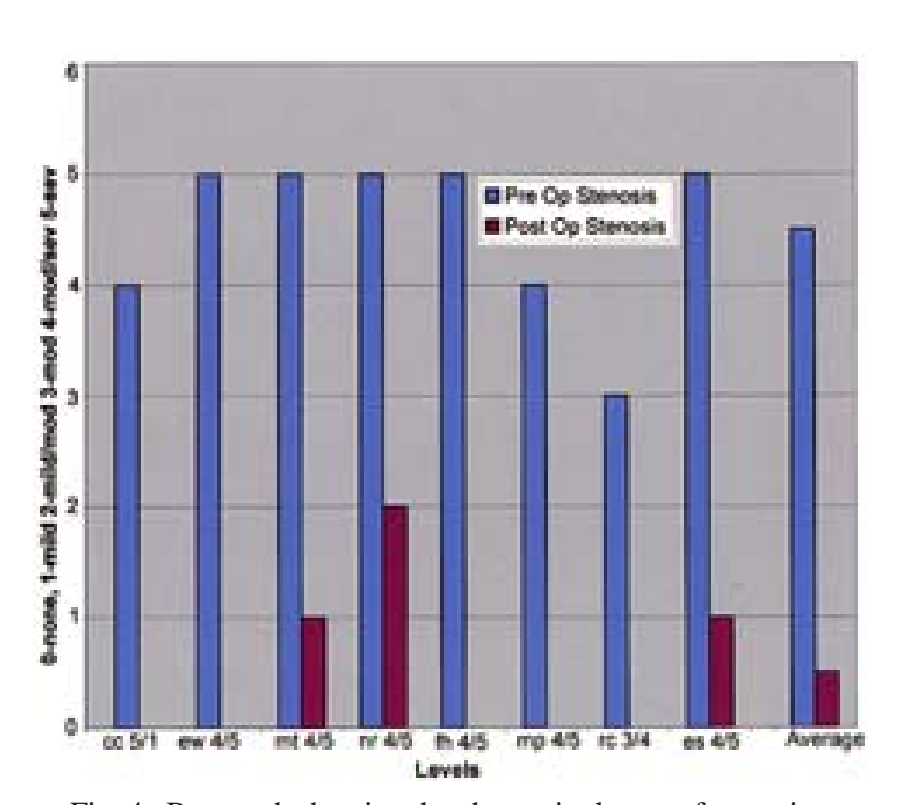

Fig. 4. Bar graph showing the change in degree of stenosis. 


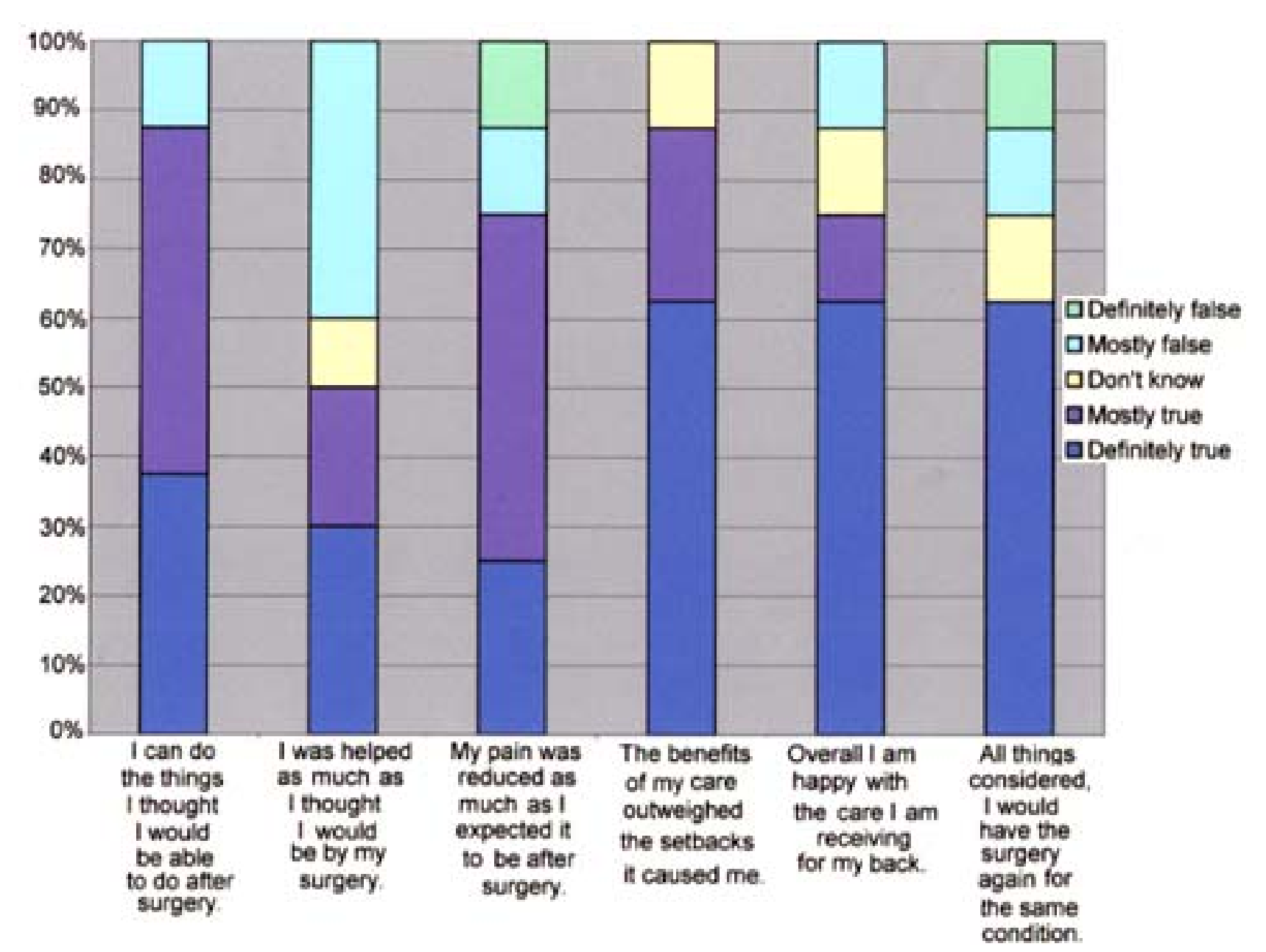

Fig. 5. Bar graph demonstrating patient satisfaction postoperatively.

ditional benefit of a minimally invasive procedure may be reduction of postoperative pain and disability and a decrease in hospital lengths of stay and thereby treatment costs.

The biomechanics of the normal spine have been extensively studied. The tendency for anterior subluxation to occur is resisted by multiple spinal elements. The facet joints have been shown to resist $33 \%$ of the shear forces, with the disc resisting 67\%. ${ }^{1}$ The supra- and interspinous ligaments resist $19 \%$ of flexion forces, with the facet capsular ligaments resisting 39\% and the disc resisting 29\%. ${ }^{1,2}$ Adams and Hutton ${ }^{1}$ have also suggested that the muscular attachments to the posterior arch and the insertions of the muscular slips on the facet capsule brace the facets, improving their ability to resist displacement. The muscular and truncal soft-tissue contributions to flexion resistance are critical because the trunk-induced force exerted on the spine in flexion is greater by twofold than that required to injure the facet joints, ${ }^{1,2}$ which would fail if unaided by other supporting tissues. The supra/intraspinous ligamentous complex has the greatest mechanical advantage because it is farthest from the axis of rotation. It is also the first to fail in flexion. ${ }^{1}$ In an anatomical study Rissanen ${ }^{21}$ found that $20 \%$ of adult spines sustained ruptures of the ligament complex and many specimens had tears in the attachments to the spinous processes. This is an anatomical verification of the biomechanical results. ${ }^{2}$

Cusick, et al., ${ }^{6}$ have conducted a biomechanical study in which they sequentially sectioned the posterior ligaments and facets and used a two-motion segment model. They noted the importance of the intact motion segment in stabilizing the altered segment, which more closely simulated the clinical situation than a single motion segment model. Although other authors of biomechanical studies have shown that the posterior ligaments make only a small contribution to overall stability, once the facet joints and the facet capsule have been altered surgically the relative contribution of the posterior ligaments to stability probably increases. ${ }^{6}$ The importance of the supra/interspinous ligament complex was emphasized. They progressively surgically altered the lumbar facet complex and found that the effects were controllable until the supra/interspinous ligaments and associated residual tendinous, midline muscle, and fascial attachments were violated. Their experimental model closely mimicked the postsurgical status evident in patients, and it stressed the contribution of the entire posterior musculoligamentous complex in resisting development of postoperative spondylolisthesis. Nevertheless, the rationale that saving as many of these elements as possible during the decompression may limit the 


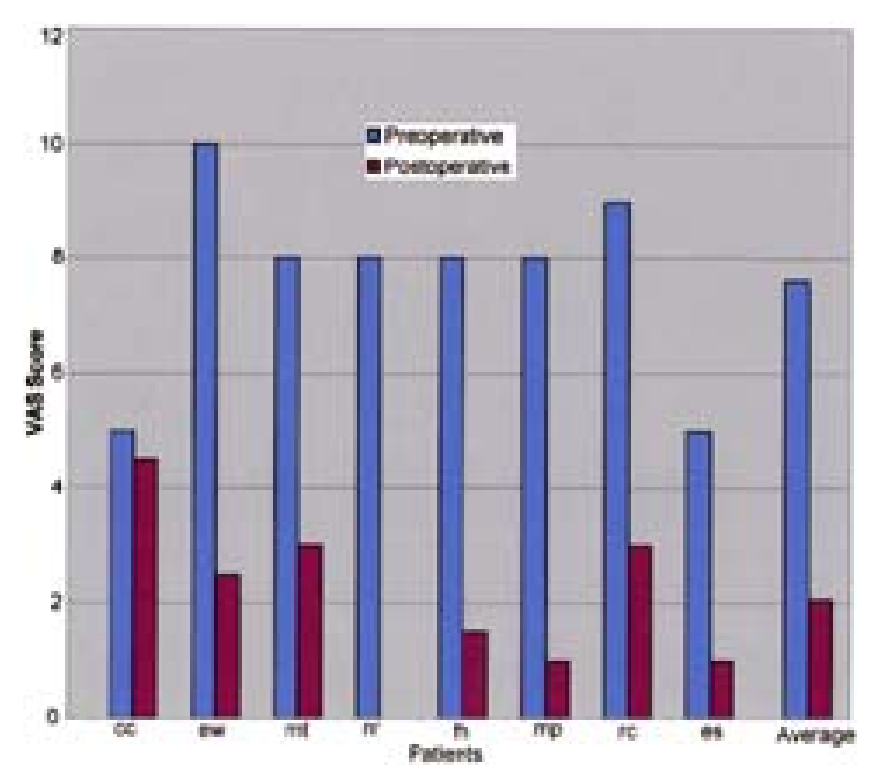

Fig. 6. Bar graph demonstrating change in VAS pain scores.

development of iatrogenic spondylolisthesis remains controversial. $3,9,19,20,29$

Spondylolisthesis has been regarded as a contraindication to simple decompressive surgery, with many authors believing that concomitant fusion is indicated. ${ }^{3,11,13,15}$ The incidence of postoperative spondylolisthesis has been reported to range from 0 to $100 \%$, with the common range being 10 to $12 \% .{ }^{13}$ Johnsson, et al., ${ }^{15}$ found a $56 \%$ incidence of increased postoperative subluxation in their patients in whom preoperative spondylolisthesis was documented. In their patients with degenerative spinal stenosis they reported a postoperative subluxation rate of $32 \%$. Mullin, et al., ${ }^{17}$ have found that patients with postoperative spinal instability tended to fair more poorly clinically with a lower ambulatory status. In our small group of patients, in those with preoperative spondylolisthesis increased postoperative subluxation did not occur. This may be the result of increased preservation of normal osseous, ligamentous, and muscular architecture when a minimally invasive procedure is performed. Thomas, et al., ${ }^{24}$ found no significant correlation between spondylolisthesis and poor results. Although our population size was small, $81 \%$ with spondylolisthesis reported that they would definitely undergo surgery again for the same condition. Their mean VAS pain score was 7.6 preoperatively and 2 postoperatively.

Minimally invasive procedures have increasing application in spinal surgery. Our results demonstrate the feasibility of performing unilateral approach minimally invasive bilateral decompression for spinal stenosis associated with degenerative spondylolisthesis. The technique has evolved from multiple previously described techniques and is now performed in conjunction with placement of METRx-MD instrumentation. Satisfactory decompression was achieved in all patients (Fig. 4). Significant stenosis of up to $45 \%$ has been found in asymptomatic patients. ${ }^{12}$ The development of acquired spinal stenosis is an incremental process; none of our patients was symp- tomatic or without at least a moderate (third-degree) stenosis. Complete decompression may not be necessary to achieve symptomatic relief as has been previously suggested by Aryanpur and Ducker. ${ }^{3}$ Thomas, et al., ${ }^{24}$ have reported a statistically significant increase in dural sac size after laminotomy or laminectomy but no statistical relationship between the extent of decompression and clinical outcome. It may only be necessary to bring the patient below a symptomatic threshold, which in our cases appeared to be moderate stenosis, to achieve a good clinical result.

Discectomy was performed in two patients with concomitant disc herniations. There has been concern that disruption of the annulus fibrosus might increase the risk of iatrogenic spondylolisthesis. ${ }^{9}$ Hopp and Tsou ${ }^{13}$ performed disc excision in half of their 344 patients and found no relationship between this surgery and postoperative vertebral instability. In our two patients no postoperative progression of the subluxation occurred.

The procedure described herein can be accomplished with reasonable operative times (mean 92 minutes) and minimal blood loss (mean $33 \mathrm{ml}$ ). Patient discomfort related to this muscle-dilating approach is such that most patients return home the same day. Although general anesthesia was induced in all patients, which is usually the patient's preference, spinal anesthesia has been successfully used since this series was collected and can be induced when desired or medically indicated.

In a metaanalysis of the literature on spinal stenosis, Turner, et al., ${ }^{25}$ found that good to excellent surgery-related outcomes varied from 26 to $100 \%$ (mean 64\%). We defined a good outcome as one in which the patient felt, in the follow-up interview, that it was definitely or mostly true that he/she was helped by the surgery and a bad outcome as one in which the patient felt he/she did not know or felt it was mostly or definitely false that he/she was helped by the surgery. The generalizability of our outcomes is limited because this study covers a small cohort of patients and because of only short-term follow-up interview results (4-7 months). The good outcomes ranged from 63 to $88 \%$ (Fig. 5), in line with other series. The VAS pain scores decreased substantially after the surgical procedure. The mean preoperative VAS was 7.5, whereas the mean postoperative VAS score was 2 (Fig. 6).

The feasibility and success in this preliminary group of patients have encouraged us to continue with this procedure. A prospective study in a larger cohort will be necessary to answer the questions raised by this preliminary study. Does this minimally invasive surgical approach to spinal stenosis offer benefits in structural integrity, iatrogenic spondylolisthesis, operative efficiency, patient outcomes, patient satisfaction, and cost?

\section{CONCLUSIONS}

Minimally invasive bilateral decompression can be successfully performed via a unilateral approach in patients with acquired spinal stenosis associated with degenerative spondylolisthesis, in conjunction with placement of METRx-MD instrumentation. The procedure can be conducted on an outpatient basis, with reasonable operative times, minimal blood loss, and acceptable morbidity. Short-term clinical outcomes in this small series are simi- 


\section{S. Palmer, R. Turner, and R. Palmer}

lar to those reported in other series in which spinal stenosis was treated surgically.

\section{Acknowledgments}

The authors acknowledge the assistance of Azhar Qureshi M.D., $\mathrm{Ph} . \mathrm{D}$., in reviewing the statistical components of this paper. They also wish to acknowledge the technical support of Medtronic Sofamor Danek.

\section{References}

1. Adams MA, Hutton WC: The mechanical function of the lumbar apophyseal joints. Spine 8:327-330, 1983

2. Adams MA, Hutton WC, Stott JR: The resistance to flexion of the lumbar intervertebral joint. Spine 5:245-253, 1980

3. Aryanpur J, Ducker T: Multilevel lumbar laminotomies: an alternative to laminectomy in the treatment of lumbar stenosis. Neurosurgery 26:429-433, 1990

4. Bailey P, Casamajor L: Osteo-arthritis of the spine as a cause of compression of the spinal cord and its roots. J Nerv Ment Dis 38:588-609, 1911

5. Choy DS, Case RE, Fielding W, et al: Percutaneous laser nucleolysis of lumbar disks. N Engl J Med 317:771-772, 1987

6. Cusick JF, Yoganandan N, Pintar FA, et al: Biomechanics of sequential posterior lumbar surgical alterations. J Neurosurg 76:805-811, 1992

7. diPierro CG, Helm GA, Shaffrey CI, et al: Treatment of lumbar spinal stenosis by extensive unilateral decompression and contralateral autologous bone fusion: operative technique and results. J Neurosurg 84:166-173, 1996

8. Dirksmeier PJ, Parsons IM IV, Kang JD: Microendoscopic and open laminotomy and discectomy in lumbar disc disease. Semin Spine Surg 11:138-146, 1999

9. Fager CA: Comment on Young S, Veerapen R, O'Laoire SA: Relief of lumbar canal stenosis using multilevel subarticular fenestrations as an alternative to wide laminectomy: preliminary report. Neurosurgery 23:628-633, 1988

10. Foley KT, Smith MM: Microendoscopic discectomy. Tech Neurosurg 3:301-307, 1997

11. Herkowitz HN, Kurz LT: Degenerative lumbar spondylolisthesis with spinal stenosis. A prospective study comparing decompression with decompression and intertransverse process arthrodesis. J Bone Joint Surg Am 73:802-808, 1991

12. Hitselburger WE, Witten RM: Abnormal myelograms in asymptomatic patients. J Neurosurg 28:204-206, 1968

13. Hopp E, Tsou PM: Postdecompression lumbar instability. Clin Orthop 227:143-151, 1988

14. Jane JA Sr, Jane JA Jr, Helm GA, et al: Acquired lumbar spinal stenosis. Clin Neurosurg 43:275-299, 1996
15. Johnsson KE, Redlund-Johnell I, Uden A, et al: Preoperative and postoperative instability in lumbar stenosis. Spine 14: 591-593, 1989

16. Kambin P, O'Brien E, Zhou L, et al: Arthroscopic microdiscectomy and selective fragmentectomy. Clin Orthop 347: 150-167, 1998

17. Mullin BB, Rea GL, Irsik R, et al: The effect of postlaminectomy spinal instability on the outcome of lumbar spinal stenosis patients. J Spinal Disord 9:107-116, 1996

18. Nakai O, Ookawa A, Yamaura I: Long-term roentgenographic and functional changes in patients who were treated with wide fenestration for central lumbar stenosis. J Bone Joint Surg Am 73:1184-1191, 1991

19. Onik G, Mooney V, Maroon JC, et al: Automated percutaneous discectomy: a prospective multi-institutional study. Neurosurgery 26:228-233, 1990

20. Poletti CE: Central lumbar stenosis caused by ligamentum flavum: unilateral laminotomy for bilateral ligamentectomy: preliminary report of two cases. Neurosurgery 37:343-347, 1995

21. Rissanen PM: The surgical anatomy and pathology of the supraspinous and interspinous ligaments of the lumbar spine with special reference to ligament ruptures. Acta Orthop Scand Suppl 46:1-100, 1960

22. Sachs B, Fraenkel J: Progressive ankylotic rigidity of the spine (spondylose rhizomelique). J Nerv Ment Dis 27:1-15, 1900

23. Smith L: Enzyme dissolution of the nucleus pulposus in humans. JAMA 187:137-140, 1964

24. Thomas NW, Rea GL, Pikul BK, et al: Quantitative outcome and radiographic comparisons between laminectomy and laminotomy in the treatment of acquired lumbar stenosis. Neurosurgery 41:567-575, 1997

25. Turner JA, Ersek M, Herron L, et al: Surgery for lumbar spinal stenosis. Attempted meta-analysis of the literature. Spine 17: $1-8,1992$

26. Verbiest H: Results of surgical treatment of idiopathic developmental stenosis of the lumbar vertebral canal. A review of twenty-seven years' experience. J Bone Joint Surg Br 59: 181-188, 1977

27. Young S, Veerapen R, O'Laoire SA: Relief of lumbar canal stenosis using multilevel subarticular fenestrations as an alternative to wide laminectomy: preliminary report. Neurosurgery 23:628-633, 1988

Manuscript received May 17, 2002.

Accepted in final form June 18, 2002.

Dr. Palmer acts as a consultant for Medtronic Sofamor Danek.

Address reprint requests to: Sylvain Palmer, M.D., 26732 Crown

Valley Parkway, Suite 561, Mission Viejo, California 92651. email: sylvainpalmer@home.com. 